\title{
Relating linear vibrational spectroscopy to condensed-phase hydrogen-bonded structures: Liquid-to-supercritical water
}

\author{
Alexander Kandratsenka, ${ }^{1}$ Dirk Schwarzer, ${ }^{1, a)}$ and Peter Vöhringer ${ }^{2, a)}$ \\ ${ }^{1}$ AG Reaktionsdynamik, Max-Planck-Institut für biophysikalische Chemie, Am Faßberg 11, \\ 37077 Göttingen, Germany \\ ${ }^{2}$ Abteilung für Molekulare Physikalische Chemie, Institut für Physikalische und Theoretische Chemie, \\ Rheinische Friedrich-Wilhelms-Universität, Wegelerstraße 12, 53115 Bonn, Germany
}

(Received 10 April 2008; accepted 23 May 2008; published online 24 June 2008)

\begin{abstract}
The pressure and temperature-dependent linear absorption spectrum of partially deuterated water HOD dissolved in heavy water $\mathrm{D}_{2} \mathrm{O}$ was measured in the $\mathrm{OH}$-stretching spectral region. The temperature was varied in the interval of $298 \mathrm{~K} \leqslant T \leqslant 700 \mathrm{~K}$ while the density was changed within the range of $12 \mathrm{~mol} / l \leqslant \rho \leqslant 58 \mathrm{~mol} / l$ corresponding to the liquid and the supercritical phases of the fluid solution. The spectra were analyzed in terms of the temperature and density dependent frequency of maximal absorbance $\widetilde{\nu}_{\max }(T, \rho)$ and their full widths at half maximum $\Delta \widetilde{\nu}(T, \rho)$. In parallel, molecular dynamics simulations of the fluid solution were carried out to obtain the average nearest neighbor $\mathrm{O}-\mathrm{O}$ distance $\left\langle r_{\mathrm{OO}}^{(1)}\right\rangle(T, \rho)$ and its dispersion $\left\langle\Delta r_{\mathrm{OO}}^{(1)}\right\rangle(T, \rho)$ at any state point $(T, \rho)$ for which an absorption spectrum was recorded. A correlation is presented between the experimental spectroscopic quantities $\widetilde{\nu}_{\max }(T, \rho)$ and $\Delta \widetilde{\nu}(T, \rho)$ on the one hand and the local structural quantities $\left\langle r_{\mathrm{OO}}^{(1)}\right\rangle(T, \rho)$ and $\left\langle\Delta r_{\mathrm{OO}}^{(1)}\right\rangle(T, \rho)$ on the other. This intuitive correlation can be used as a critical test for future perturbational simulations of the $\mathrm{OH}$-stretching frequency shifts with hydrogen-bond geometry. Finally, a connection is made to the average hydrogen-bond connectivity in the fluid via the temperature and density dependent dielectric constant of water. (c) 2008 American Institute of Physics. [DOI: 10.1063/1.2945896]
\end{abstract}

\section{INTRODUCTION}

Noncovalent intermolecular interactions and their relationship to the thermally induced many-body positional and orientational molecular dynamics are central issues for a comprehensive understanding of the physicochemical properties of disordered condensed-phase systems. Intermolecular hydrogen-bonding phenomena ${ }^{1,2}$ in bulk liquids are particularly intriguing because they give rise to unexpected thermodynamic properties and transport parameters. The most prominent example for hydrogen-bonded liquids is water ${ }^{3}$ with its frequently cited anomalies. Yet, despite being a wellestablished concept in the chemical, physical, and the life sciences, an unambiguous and nonarbitrary definition ${ }^{4-9}$ of a hydrogen-bond (H-bond) is still lacking.

Structural information about hydrogen bonding in bulk liquid water may be inferred from diffraction studies. X-ray and neutron scatterings can be used to retrieve the various radial pair distribution functions and therefore to construct an average image of the structural packing of molecules within the liquid. ${ }^{10-14}$ Combined with theoretical studies, ${ }^{5,6,15-19}$ a local ordering with a quasitetrahedral coordination can be derived for liquid $\mathrm{H}_{2} \mathrm{O}$ that exhibits a substantial variance of both, the interparticle distances, and the intermolecular orientations. From molecular dynamics (MD) calculations on liquid water, a random network of hydrogen

\footnotetext{
${ }^{a)}$ Author to whom correspondence should be addressed. Electronic mails: dschwar@gwdg.de and p.voehringer@uni-bonn.de.
}

bridges can then be identified whose spatial dimension depends on the geometric and energetic criteria invoked in identifying H-bonded configurations. ${ }^{17,20}$

On the other hand, dynamical information about hydrogen bonding in bulk liquid water may be inferred from vibrational spectroscopies. Raman-induced optical Kerreffect spectroscopy ${ }^{21-23}$ and far-infrared absorption spectroscopies ${ }^{24}$ were used to expose the linear lineshapes of intermolecular modes of liquid $\mathrm{H}_{2} \mathrm{O}$ that modulate directly local hydrogen-bond geometries such as hindered translational and librational degrees of freedom.

More recently, time resolved mid-infrared (MIR) spectroscopy and its two-dimensional variants with femtosecond laser pulses have proven particularly valuable in elucidating the mechanisms and time scales for hydrogen-bond dynamics. ${ }^{25-32}$ In contrast to the aforementioned spectroscopic techniques, femtosecond MIR-spectroscopy uses intramolecular vibrations of the water molecules as spectroscopic probes of their local hydrogen-bonded environment. Configurational fluctuations of a hydrogen-bond to a neighboring particle modulate the resonance frequency of the intramolecular $\mathrm{OH}$-modes of a tagged water molecule thereby forcing it to spectrally diffuse and to contribute to the spectral shape of the apparent vibrational resonance of the liquid ensemble.

Quite intuitively, the hydroxyl stretching vibrator should serve as a particularly sensitive marker vibration for structural dynamics of the hydrogen-bond to which it is directly linked according to $\mathrm{O}-\mathrm{H} \cdots \mathrm{O}$. This idea has led $\mathrm{Novak}^{33}$ to 
devise a classification of hydrogen-bonds on solids based on a correlation of spectroscopic and crystallographic data accumulated from a large body of crystalline molecular substances. According to this scheme, weak hydrogen-bonds are those that lead to $\mathrm{OH}$-stretching resonance frequencies $\widetilde{\nu}_{\mathrm{OH}}$ in excess of $3200 \mathrm{~cm}^{-1}$ and oxygen-oxygen distances of $\sim 2.70 \AA$. In contrast, strong hydrogen-bonds are identified as those that result in $\mathrm{OH}$-stretching frequencies of $2700 \mathrm{~cm}^{-1}$ and below while the $\mathrm{O}-\mathrm{O}$ distance is smaller than $2.60 \AA$ A. For weakly hydrogen-bonded water molecules Mikenda found a correlation between $\mathrm{OD}(\mathrm{H})$-stretching frequency and hydrogen-bond length by compilation of spectroscopic and structural data of solid hydrates. ${ }^{34}$

It is this empirical classification from solid state chemistry that has been used exhaustively to interpret recent timeresolved spectroscopic studies on the vibrational dynamics and spectral diffusion within hydrogen-bonded liquids. ${ }^{35,36}$ Taking the relative displacement of the two oxygen atoms involved in the hydrogen-bond as a direct measure for the length of the $\mathrm{H}$-bridge it follows that $\mathrm{OH}$ vibrators engaged in short and strong hydrogen-bonds absorb in the blue spectral parts of the ensemble $\mathrm{OH}$-stretching resonance while hydroxyl oscillators involved in long and weak H-bonds absorb in the red spectral edge.

In this paper, we wish to derive an alternative correlation between hydrogen-bond distance and $\mathrm{OH}$ stretching frequency that relies on thermodynamic and linear spectroscopic data of liquid water itself rather than on an empirical correlation based on solid hydrates or even worse, a collection of solid-state data of chemical compounds that are entirely distinct from $\mathrm{H}_{2} \mathrm{O}$. Structural information is obtained from independent molecular dynamics simulations of the liquid and provides the conceptual link between bulk spectroscopic and thermodynamic data on the one hand and local hydrogen-bonded structures on the other.

\section{EXPERIMENTAL}

Linear absorption spectra as a function of temperature $T$ and pressure $p$ were recorded with a Fourier-transform infrared spectrometer (Perkin-Elmer, Spectrum BX) with a spectral resolution of $0.5 \mathrm{~cm}^{-1}$. The fluid sample was contained in a high pressure thermostatic optical cell that was equipped with a pair of sapphire windows for spectroscopic measurements in transmission mode. The clear aperture of the sample cell was $2.5 \mathrm{~mm}$ while its optical path length could be adjusted between a few tens of microns to about $0.5 \mathrm{~mm}$ by changing the distance between the two sapphire windows. For the experiments reported herein, the pressure was varied between 1 and 1500 bars by means of a manual hydrostatic pump and was recorded with a pressure gauge with an accuracy of $1 \%$. The sample temperature was controlled between room temperature and $700 \mathrm{~K}$ with heating cartridges embedded in the cell body and was measured via a thermocouple in direct contact with the liquid with an accuracy of $\pm 1 \mathrm{~K}$. The sample consisted of mixtures of $\mathrm{H}_{2} \mathrm{O}$ and $\mathrm{D}_{2} \mathrm{O}$ corresponding, due to rapid isotope exchange, to solutions of the mixed isotopomer $\mathrm{HOD}$ (the solute) in $\mathrm{D}_{2} \mathrm{O}$ with a concentration of $2 \%$. The concentration of the solute is low enough to avoid

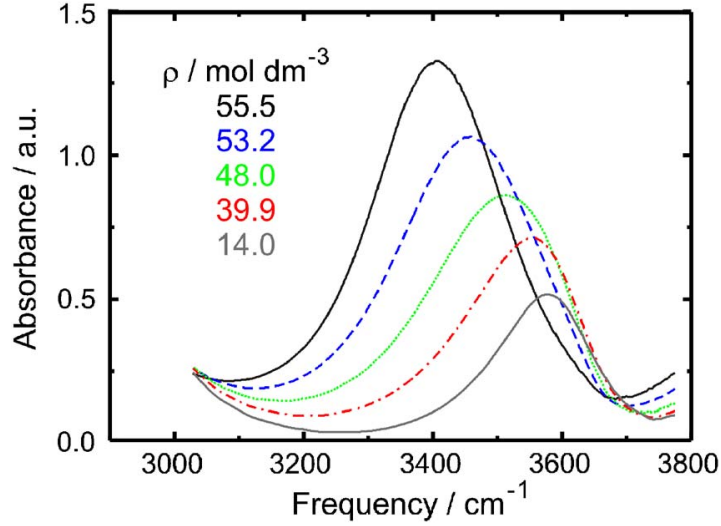

FIG. 1. (Color online) Temperature and density dependent linear absorption spectra in the $\mathrm{OH}$-stretching region of $2 \mathrm{~mol} \% \mathrm{HOD}$ in liquid-tosupercritical $\mathrm{D}_{2} \mathrm{O}$. The state points correspond to $296 \mathrm{~K}$ and 1 bar (black), $376 \mathrm{~K}$ and 2 bar (blue), $472 \mathrm{~K}$ and 17 bar (green), $570 \mathrm{~K}$ and 90 bar (red), and $670 \mathrm{~K}$ and 350 bar (gray). The legend indicates the density.

any contributions arising from cooperative $\mathrm{HO}-\mathrm{HO}$ resonance interactions. The pressure and temperature-dependent density $\rho(p, T)$ of the sample as well as its dielectric constant $\varepsilon(p, T)$ was accessible from the PROPATH computer package for thermophysical properties of fluids. ${ }^{37}$

\section{RESULTS AND DISCUSSION}

The influence of decreasing density and increasing temperature on the appearance of the linear absorption spectrum of HOD in heavy water is illustrated in Fig. 1. All spectra were recorded at the same solute concentration of $2 \%$ and at a constant optical path length of $0.2 \mathrm{~mm}$. Because the density of the sample varies with temperature and pressure, the solute's molar concentration is also a function of $T$ and $p$. Therefore, the experimentally determined raw spectra recorded at the thermodynamic state points $(T, p)$ had to be multiplied by the factor $\rho(p, T) / \rho(1$ bar, $298 \mathrm{~K})$ to obtain the pressure and temperature-dependent relative extinction coefficient of the solute HOD dissolved in $\mathrm{D}_{2} \mathrm{O}$. It is obvious that with decreasing density the absorption spectrum of the $\mathrm{OH}-$ stretching mode shifts to higher frequencies to gradually approach the spectral position of the gas phase resonance. At the same time, the absorption band loses spectral bandwidth and overall intensity upon lowering the liquid density. These findings are in complete agreement with the pioneering studies of Franck et al. ${ }^{38}$ on the OD stretching vibration of dilute $\mathrm{HOD}$ in $\mathrm{H}_{2} \mathrm{O}$ and later experiments proving the existence of hydrogen-bonds in supercritical water above $\sim 50$ bars. $^{39,40}$

The spectral shift can be interpreted as follows: Upon lowering the density the average interparticle distance and hence the mean oxygen-oxygen distance increases thereby raising the fraction of weak hydrogen bonds in the random water network at the expense of strong hydrogen-bonds. The average interparticle $\mathrm{O}-\mathrm{O}$ distance is denoted $\left\langle r_{\mathrm{OO}}\right\rangle_{\text {bulk }}$ which can be calculated from the bulk density $\rho$ of the fluid solution according to $\left(\rho N_{A}\right)^{-1 / 3}$ where $N_{A}$ is Avogadro's number.

To characterize the strength of the hydrogen-bond network in the fluid the experimentally determined frequency $\widetilde{\nu}_{\max }$ of maximal absorbance of the $\mathrm{OH}$-stretching resonance of HOD (see Fig. 1) can be correlated with the mean inter- 


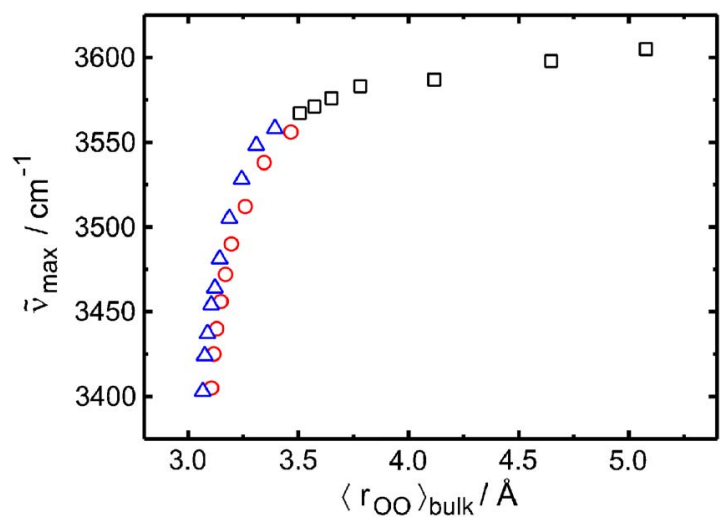

FIG. 2. (Color online) Frequency of maximal linear absorption in the $\mathrm{OH}$ stretching region of HOD in liquid-to-supercritical $\mathrm{D}_{2} \mathrm{O}$ as a function of the mean interparticle $\mathrm{O}-\mathrm{O}$ distance calculated from the pressure and temperature-dependent bulk density. The thermodynamic conditions correspond to $T=696 \mathrm{~K}$ and $p=320-1260$ bar (black squares), $T=297-573 \mathrm{~K}$ and $p=p_{\text {sat }}(T)$, where $p_{\text {sat }}(T)$ is the temperature-dependent saturation pressure (red circles), and $T=297-623 \mathrm{~K}$ and $p=1000$ bars (blue triangles).

particle O-O distance obtained from the bulk density. Such a correlation is displayed in Fig. 2. As expected, the absorption maximum shifts towards lower frequencies with decreasing interparticle distance and hence with a growing fraction of strong hydrogen bonds within the network. Qualitatively, this correlation resembles very much the correlation between $\mathrm{OH}$-stretching frequency and $\mathrm{O}-\mathrm{O}$ distance from $\mathrm{x}$-ray and neutron diffraction proposed by Novak ${ }^{33}$ for molecular crystals and Mikenda ${ }^{34}$ for water in solid hydrates. However, compared to the data base of water in solid hydrates from Ref. 34, the dispersion of vibrational frequencies in liquidto-supercritical water is smaller. On the other hand, the range of interparticle $\mathrm{O}-\mathrm{O}$ distances accessible to liquid-tosupercritical $\mathrm{H}_{2} \mathrm{O}$ is much wider as compared to the molecular lattices considered by Mikenda.

Unfortunately, the average interparticle $\mathrm{O}-\mathrm{O}$ distance obtained from the bulk density is not the relevant structural quantity that is required to characterize the average hydrogen-bond geometry at a given thermodynamic state point $(p, T)$. This is because the bulk density is not at all a local density around a tagged central water molecule. It is this latter quantity only that can specify the hydrogen-bond connectivity because it contains information about the distance of a central particle to its nearest neighbors, its next nearest neighbors and so forth, not all of which may be hydrogen-bonded to it.

In this work, we obtain the information about the local density as a function of pressure and temperature from independent classical molecular dynamics simulations. ${ }^{41}$ The MD simulations were performed with 108 water molecules in a cubic simulation box with periodic boundary conditions using the MOLDY program. ${ }^{42}$ The extended single point charge (SPC/E) model of water was adopted to describe the rigid water geometry and intermolecular interactions. ${ }^{43}$ The SPC/E water model was chosen because it is known to predict rather well the temperature and pressure dependent number density in liquid-to-supercritical water while reproducing at the same time the structural properties from ambient to supercritical conditions. ${ }^{12}$ Long-range Coulomb interactions were handled

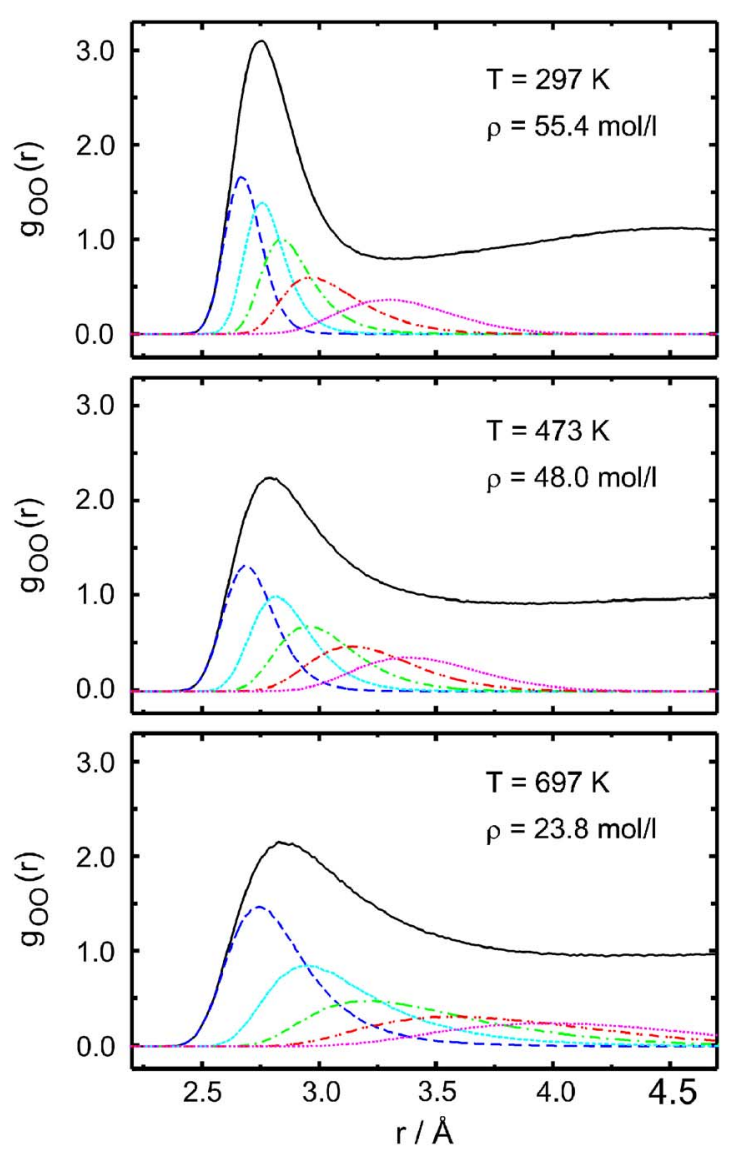

FIG. 3. (Color online) O-O pair correlation functions for various thermodynamic conditions and individual $\mathrm{O}-\mathrm{O}$ pair correlation functions $g_{\mathrm{OO}}^{(1)}(r)$ (nearest $\mathrm{O}$, blue), $g_{\mathrm{OO}}^{(2)}(r)$ (next nearest $\mathrm{O}$, cyan), $g_{\mathrm{OO}}^{(3)}(r)$ (third nearest $\mathrm{O}$, green), $g_{\mathrm{OO}}^{(4)}(r)$ (fourth nearest $\mathrm{O}$, red), and $g_{\mathrm{OO}}^{(5)}(r)$ (fifth nearest $\mathrm{O}$, magenta).

by applying the standard Ewald summation technique. The dimension of the simulation box was chosen to yield the desired density at the given state point. Calculations were performed in the NVT ensemble employing a Nosé-Hoover thermostat. The equations of motion were integrated by means of the Refson-Beeman algorithm with a time step of $0.5 \mathrm{fs}$. After an equilibration period of $20 \mathrm{ps}$, the simulation time was $1 \mathrm{~ns}$. Oxygen-oxygen pair correlation functions $g_{\text {OO }}(r)$ were calculated at every state point used to measure the mid-infrared absorption spectrum.

Typical oxygen-oxygen pair correlation functions $g_{\mathrm{OO}}(r)$ are reproduced in Fig. 3. Following the work by Kumar et $a l .{ }^{17}$ on hydrogen-bond definitions and dynamics in liquid water, $g_{\mathrm{OO}}(r)$ can be decomposed into individual $\mathrm{O}-\mathrm{O}$ pair correlation functions $g_{\mathrm{OO}}^{(i)}(r)$ that contain contributions arising from the nearest neighbor oxygen atom, $i=1$, the next nearest neighbor oxygen atom, $i=2$, and so forth, to a given hydrogen atom of a selected central water molecule. By identifying the nearest and next nearest neighbor oxygens to the hydrogen atom of a central $\mathrm{H}_{2} \mathrm{O}$ particle, the analysis is biased to count preferentially the $\mathrm{O}$-atoms of those water molecules to which the central water is already properly oriented for hydrogen-bond formation. As pointed out in Ref. 17 , the widths of these individual pair correlation functions and the location of their maxima are a measure of the structural disorder in the fluid. 


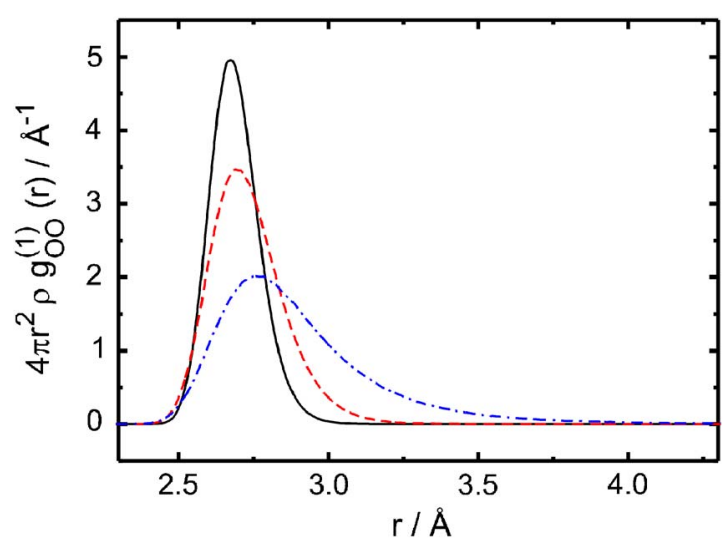

FIG. 4. (Color online) Nearest-neighbor contribution to the probability density for different thermodynamic conditions. $T=297 \mathrm{~K}$ and $\rho=55.4 \mathrm{~mol} / 1$ (black), $T=473 \mathrm{~K}$ and $\rho=48.0 \mathrm{~mol} / 1$ (red), and $T=697 \mathrm{~K}$ and $\rho$ $=23.8 \mathrm{~mol} / 1$ (blue).

In the following we will focus on the nearest neighbor contribution to the radial probability density $4 \pi r^{2} \cdot g_{\mathrm{OO}}^{(1)}(r)$ or the probability $4 \pi r^{2} \cdot g_{\mathrm{OO}}^{(1)}(r) d r$ for finding the oxygen atom of a nearest neighbor particle at a distance between $r$ and $r$ $+d r$ from the oxygen atom of a central water molecule. Note that all integrals $\int_{0}^{\infty} 4 \pi r^{2} \cdot g_{\mathrm{OO}}^{(i)}(r) d r$ must be unity by definition. Representative nearest neighbor probability densities $4 \pi r^{2} \cdot g_{\mathrm{OO}}^{(1)}(r)$ for different thermodynamic conditions are reproduced in Fig. 4. Clearly, with decreasing density of the solution, the curves shift towards larger values of $r$ demonstrating that the nearest neighbor $\mathrm{O}-\mathrm{O}$ distance increases upon lowering the total number of molecules per unit volume as expected. Furthermore, the distributions become increasingly broader and more asymmetric with decreasing density, which can be associated again with an increasing disorder as the interparticle distance becomes larger.

Having defined the nearest neighbor probability density, the expectation value for the nearest neighbor $\mathrm{O}-\mathrm{O}$ distance can now be calculated,

$$
\left\langle r_{\mathrm{OO}}^{(1)}\right\rangle=\int_{0}^{\infty} r g_{\mathrm{OO}}^{(1)}(r) 4 \pi r^{2} d r
$$

easily at each state point, as well as the $\mathrm{O}-\mathrm{O}$ distance $r_{\mathrm{OO} \text {,max }}^{(1)}$ where the nearest neighbor probability density is maximal.

We can now correlate an experimentally accessible spectroscopic parameter with a structural parameter that is accessible from the MD simulations on SPC/E water provided both quantities were determined for the same thermodynamic state point of the fluid solution. The spectroscopic parameter is the density and temperature-dependent frequency of maximal absorbance in the $\mathrm{OH}$-stretching region $\widetilde{\nu}_{\max }(\rho, T)$, while the density and temperature-dependent $\mathrm{O}-\mathrm{O}$ distance of maximal nearest neighbor probability density $r_{\mathrm{OO}, \max }^{(1)}(\rho, T)$ may be chosen as the structural parameter. Such a correlation is reproduced in Fig. 5.

To this correlation, we can also add the full width at half maximum $\Delta \widetilde{\nu}_{\max }(\rho, T)$ of the experimentally determined $\mathrm{OH}-$ stretching absorption band as a measure for the dispersion of the hydroxyl resonance frequency in the fluid ensemble (see vertical bars in Fig. 5). Similarly, we can quantify the disper-

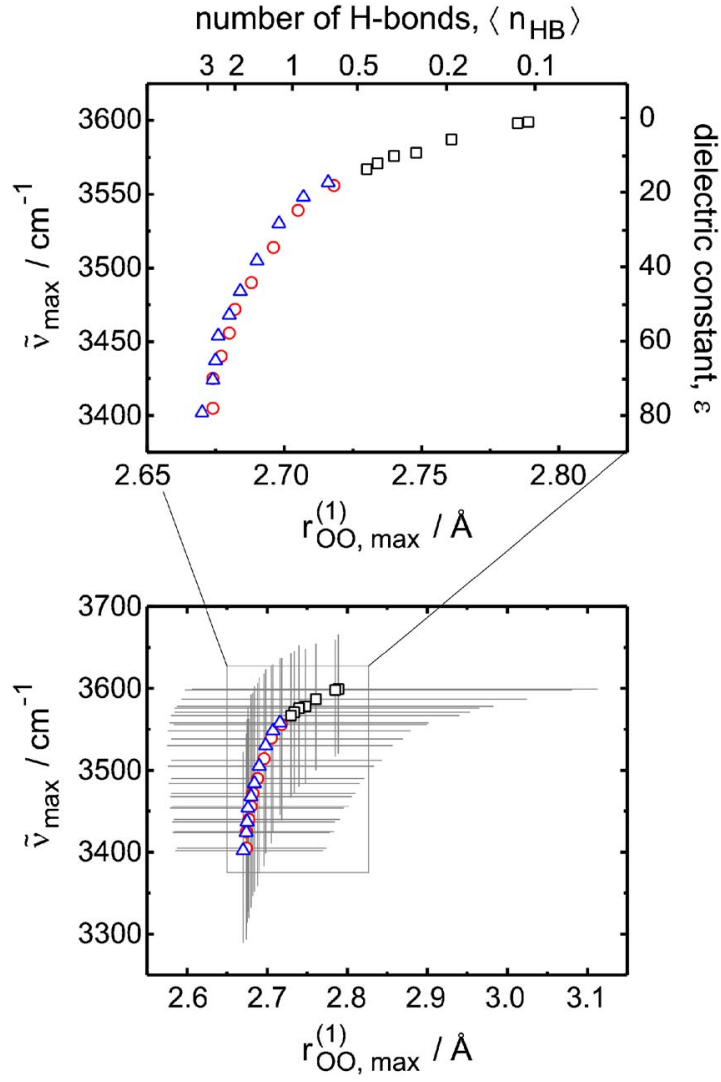

FIG. 5. (Color online) Upper panel: Frequency of maximal OH-stretching absorbance of $\mathrm{HOD}$ in $\mathrm{D}_{2} \mathrm{O}$ as a function of the $\mathrm{O}-\mathrm{O}$ distance of maximal nearest neighbor probability density. The right axis contains the temperature and density dependent dielectric constant of the fluid solution and the upper axis contains the average number of hydrogen-bonds per water molecule. The thermodynamic conditions correspond to $T=696 \mathrm{~K}$ and $p$ $=320-1260$ bars (black squares), $T=297-573 \mathrm{~K}$ and $p=p_{\text {sat }}(T)$, where $p_{\text {sat }}(T)$ is the temperature-dependent saturation pressure (red circles), and $T=297-623 \mathrm{~K}$ and $p=1000$ bars (blue triangles). Lower panel: Symbols as above. Vertical bars correspond to the full width at half maximum, $\Delta \widetilde{\nu}_{\max }$ $(\rho, T)$ of the $\mathrm{OH}$-stretching band and the horizontal bars reproduce the full width at half maximum $\left\langle\Delta r_{\mathrm{OO}}^{(1)}\right\rangle$, of the nearest neighbor O-O probability density.

sion of the nearest-neighbor $\mathrm{O}-\mathrm{O}$ distances from the probability densities shown in Fig. 4 by extracting their full widths at half maximum $\left\langle\Delta r_{\mathrm{OO}}^{(1)}\right\rangle$ (see horizontal bars in Fig. $5)$.

An equivalent correlation plot of the density and temperature-dependent frequency of maximal absorbance in the $\mathrm{OH}$-stretching region $\widetilde{\nu}_{\max }(\rho, T)$ as a function of the density and temperature-dependent expectation value for the nearest neighbor $\mathrm{O}-\mathrm{O}$ distance $\left\langle r_{\mathrm{OO}}^{(1)}\right\rangle(\rho, T)$ is reproduced in Fig. 6. Notice that the bars representing the $\mathrm{OH}$-frequency dispersion and the $\mathrm{O}-\mathrm{O}$ distance dispersion are asymmetric with respect to the data tuples $\widetilde{\nu}_{\max }(\rho, T)$ vs $r_{\mathrm{OO} \text {, max }}^{(1)}(\rho, T)$ [and $\widetilde{\nu}_{\max }(\rho, T)$ vs $\left\langle r_{\mathrm{OO}}^{(1)}\right\rangle(\rho, T)$ ] because of the intrinsic asymmetry of both the $\mathrm{OH}$-stretching absorption band and the nearest neighbor oxygen-oxygen probability density. This becomes especially apparent for the low density data. The data (Fig. 6) can be fitted to the empirical stretched exponential function, 


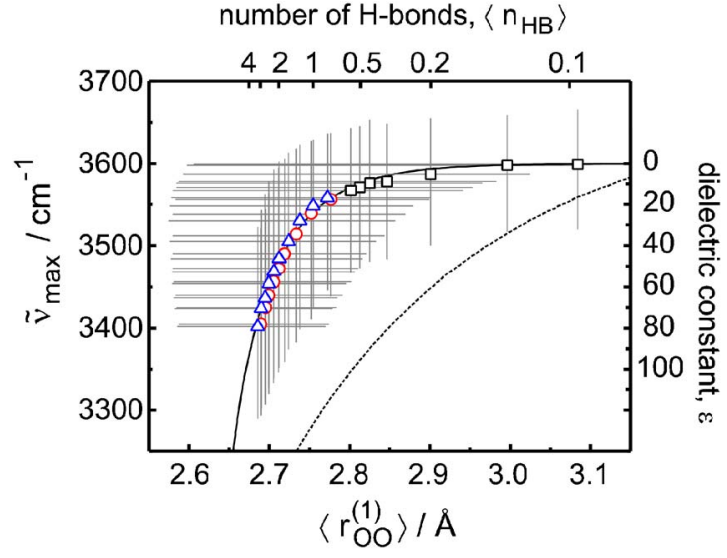

FIG. 6. (Color online) Frequency of maximal OH-stretching absorbance of $\mathrm{HOD}$ in $\mathrm{D}_{2} \mathrm{O}$ as a function of the expectation value for the nearest neighbor $\mathrm{O}-\mathrm{O}$ distance (symbols as in Fig. 5). The solid curve corresponds to the empirical fit according to Eq. (2) while the dashed curve represents the O-O distance dependence for water molecules embedded in solid hydrates from Ref. 34.

$$
\begin{aligned}
\tilde{\nu}_{\max }\left(\left\langle r_{\mathrm{OO}}^{(1)}\right\rangle\right)= & \widetilde{\nu}_{\max }(\infty)-400 \mathrm{~cm}^{-1} \\
& \times\left\{\exp \left(-\frac{\left\langle r_{\mathrm{OO}}^{(1)}\right\rangle-2.65 \AA}{0.05 \AA}\right)^{0.85}\right\},
\end{aligned}
$$

with a frequency at infinite nearest-neighbor O-O distance of $\widetilde{\nu}_{\max }(\infty)=3600 \mathrm{~cm}^{-1}$. It is interesting to note that this value does not agree with the $\mathrm{OH}$-stretching frequency derived from the spectral position of its $Q$-branch in the dilute gas phase. A similar finding was reported by Franck and Roth ${ }^{38}$ for the absorption band of the OD-stretching vibration of $\mathrm{HOD}$ in liquid-to-supercritical $\mathrm{H}_{2} \mathrm{O}$, which converges with decreasing density onto the $P$-branch. Franck and Roth suggested that $\widetilde{\nu}_{\max }(\infty)$ might reflect the combined influence of the surrounding polar water molecules on the hydroxyl oscillator when hydrogen-bonding interactions are turned off.

The dashed curve in Fig. 6 represents the O-O distance dependence of the $\mathrm{OH}$-stretching vibration derived from water molecules embedded in solid hydrates. ${ }^{34}$ Although the root mean squared deviation of the observed frequencies from this curve was reported to be $\sim 40 \mathrm{~cm}^{-1}$, it is quite clear that for a given distance the $\mathrm{OH}$-stretching frequency is higher in the liquid than in solid hydrates. Accordingly, on average the hydrogen-bond is weaker in the liquid than in the crystal for fixed $\mathrm{O}-\mathrm{O}$ distance. As the $\mathrm{OH}$-stretching frequency not only depends on the $\mathrm{O}-\mathrm{O}$ distance but also on the $\mathrm{O}-\mathrm{H} \cdots \mathrm{O}$ hydrogen-bond angle ${ }^{16,44}$ this discrepancy may be caused by the fact that for the solid state data preferentially more linear hydrogen-bonds have been evaluated, whereas in the liquid a broader angle distribution is sampled.

Figures 5 and 6 can be compared to typical joint probability distributions for the $\mathrm{OH}$-stretching frequency and the hydrogen-bond length that have previously been calculated for $\mathrm{HOD}$ in $\mathrm{D}_{2} \mathrm{O}$ in a semiclassical fashion. Based on the approach initially developed by Oxtoby et al., ${ }^{45}$ the groups of Hynes ${ }^{16,46}$ and Skinner ${ }^{44,47}$ calculated vibrational lineshapes from the intrinsic dynamics of water hydrogen-bonds and made the connection to vibrational lifetimes and spectral diffusion in this system. They treated the HOD solute vibra- tions quantum-mechanically while all other degrees of freedom of the system including those of the $\mathrm{D}_{2} \mathrm{O}$ bath were handled classically. The quantity most intimately linked to the $\mathrm{OH}$-stretching frequency of the solute was the solutebath coupling, whose dynamics were obtained from a classical molecular dynamics simulation.

Taking the O-O distance as a measure for the length of the hydrogen bridge, we observe qualitatively the very same features: (i) Long hydrogen-bonds are associated with larger $\mathrm{OH}$-frequencies and give rise to blue-shifted linear absorptions, (ii) short hydrogen-bonds are related to smaller vibrational frequencies and cause blue-shifted $\mathrm{OH}$-stretching absorptions, and (iii) the distance uncertainty of the correlation (corresponding to the width of the joint probability distribution in Refs. 16 and 44) grows with increasing OH-stretching resonance frequency. The fundamental difference to Refs. 16 and 44 is that the correlations presented in Figs. 5 and 6 rely on $\mathrm{OH}$-stretching frequencies whose magnitude and distance-dependence have been obtained experimentally by measuring the linear mid-infrared absorption as a function of the particle density in the fluid solution. Therefore, we view the results presented here as a suitable benchmark for future molecular-level computational studies of the hydrogen-bond dynamics in liquid water.

In previous publications, we have presented the full temperature and density dependence of the vibrational relaxation dynamics of HOD solutions in liquid-to-supercritical $\mathrm{D}_{2} \mathrm{O}$ following an initial femtosecond OH-stretching excitation. ${ }^{48,49}$ In trying to obtain an independent experimental measure for the local density of the solvent around the vibrationally excited solute, we have identified an empirical relationship between the vibrational relaxation rate coefficients and the dielectric constant $\varepsilon$ of the fluid. From MD simulations on water from ambient-to-supercritical conditions using the TIP4P-FQ model potential, Yoshii et al. ${ }^{15}$ found a linear dependence between the dielectric constant and the average number of hydrogen-bonds $\left\langle n_{\mathrm{HB}}\right\rangle$, a given water molecule is engaged in.

Being now equipped with the frequency of maximal absorbance in the $\mathrm{OH}$-stretching region $\widetilde{\nu}_{\max }(\rho, T)$ and the dielectric constant $\varepsilon(\rho, T)$ of water, both of which are observables that are experimentally determined as a function of density and temperature, it is possible to finally make the direct connection between the nearest-neighbor $\mathrm{O}-\mathrm{O}$ distance (as a measure for the hydrogen-bond geometry) and the average number of hydrogen- bonds per water molecule (as a measure of the H-bond connectivity in the fluid). This can simply be accomplished via the dependence of $\widetilde{\nu}_{\max }(\rho, T)$ on $\varepsilon(\rho, T)$, which we reported earlier in great detail. ${ }^{48,49}$ The top axes in Figs. 5 and 6 is a scale for the resultant number of $\mathrm{H}$-bonds that corresponds to a mean $\mathrm{O}-\mathrm{O}$ distance between nearest neighbors while the right axes represents the dielectric constant that gives rise to an apparent value for $\widetilde{\nu}_{\max }$ of the $\mathrm{OH}$-stretching absorption band.

Figures 5 and 6 demonstrate quite uniquely that it is entirely equivalent to discuss vibrational $\mathrm{OH}$-stretching lineshapes and time-resolved femtosecond mid-IR spectroscopies in terms of hydrogen-bond length fluctuations on the one hand or hydrogen-bond breakage and formation (i.e., 
a structural reorganization) on the other. Both views can now be translated into one another simply by inspection of the correlations displayed in Figs. 5 and 6. The quantitative reliability of these correlations hinges on the ability of the model potential used as input for the MD-simulations (here: $\mathrm{SPC} / \mathrm{E})$ to reproduce the thermodynamic $p V T$-data of the fluid and the radial pair correlation functions available from $\mathrm{x}$-ray and neutron scatterings. Obviously, there is still room for improvement but at this stage it suffices to emphasize that the difference between these two interpretations (i.e., $\mathrm{H}$-bond length fluctuations or $\mathrm{H}$-bond structural reorganization) depends obviously on the definition of a hydrogenbond-an issue most recently addressed by Kumar et al. ${ }^{17}$ quite comprehensively.

\section{CONCLUSIONS}

In summary, we have presented a correlation of the $\mathrm{OH}$ stretching frequency of water with the hydrogen-bond length based exclusively on experimental data of the temperature and density dependences of the linear mid-IR absorption spectrum of HOD in liquid-to-supercritical $\mathrm{D}_{2} \mathrm{O}$ accompanied by molecular dynamics calculations. We very much hope that this correlation will serve as an important benchmark for future simulations of the influence of hydrogen bonding on the vibrational dynamics and spectroscopy of liquid water.

\section{ACKNOWLEDGMENTS}

Financial support by the Deutsche Forschungsgemeinschaft through Grant No. VO 593/5-1 and SCHR 303/1-1 is gratefully acknowledged.

${ }^{1}$ Hydrogen Bonding — New Insights, edited by S. J. Grabowski (Springer, Amsterdam, 2006), Vol. 3.

${ }^{2}$ G. C. Pimentel and A. L. McClellan, The Hydrogen Bond (Freeman, San Francisco 1960).

${ }^{3}$ Water: A Comprehensive Treatise, edited by F. Franks (Plenum, New York, 1972).

${ }^{4}$ W. L. Jorgensen, Chem. Phys. Lett. 70, 326 (1980).

${ }^{5}$ W. L. Jorgensen, J. Chandrasekhar, J. D. Madura, R. W. Impey, and M. L. Klein, J. Chem. Phys. 79, 926 (1983).

${ }^{6}$ A. Rahman and F. H. Stillinger, J. Chem. Phys. 55, 3336 (1971).

${ }^{7}$ A. Luzar and D. Chandler, Phys. Rev. Lett. 76, 928 (1996).

${ }^{8}$ F. Sciortino and S. L. Fornili, J. Chem. Phys. 90, 2786 (1989).

${ }^{9}$ F. W. Starr, J. K. Nielsen, and H. E. Stanley, Phys. Rev. E 62, 579 (2000).

${ }^{10}$ A. H. Narten and H. A. Levy, J. Chem. Phys. 55, 2263 (1971).

${ }^{11}$ A. K. Soper, Chem. Phys. 258, 121 (2000).

${ }^{12}$ G. Hura, D. Russo, R. M. Glaeser, T. Head-Gordon, M. Krack, and M. Parrinello, Phys. Chem. Chem. Phys. 5, 1981 (2003).

${ }^{13}$ A. Botti, F. Bruni, M. A. Ricci, and A. K. Soper, J. Chem. Phys. 109,
3180 (1998).

${ }^{14}$ M.-C. Bellissent-Funel, J. Mol. Liq. 90, 313 (2001).

${ }^{15}$ N. Yoshii, S. Miura, and S. Okazaki, Chem. Phys. Lett. 345, 195 (2001).

${ }^{16}$ K. B. Møller, R. Rey, and J. T. Hynes, J. Phys. Chem. A 108, 1275 (2004).

${ }^{17}$ R. Kumar, J. R. Schmidt, and J. L. Skinner, J. Chem. Phys. 126, 204107 (2007).

${ }^{18}$ K. Laasonen, M. Sprik, and M. Parrinello, J. Chem. Phys. 99, 9080 (1993).

${ }^{19}$ P. H.-L. Sit and N. Marzari, J. Chem. Phys. 122, 204510 (2005).

${ }^{20}$ J. Marti, J. Chem. Phys. 110, 6876 (1999).

${ }^{21}$ E. W. Castner, Y. J. Chang, Y. C. Chu, and G. E. Walrafen, J. Chem. Phys. 102, 653 (1995).

${ }^{22}$ K. Winkler, J. Lindner, and P. Vöhringer, Phys. Chem. Chem. Phys. 4, 2144 (2002).

${ }^{23}$ K. Winkler, J. Lindner, and P. Vöhringer, J. Chem. Phys. 113, 4674 (2000).

${ }^{24}$ C. Rønne and S. R. Keiding, J. Mol. Liq. 101, 199 (2002).

${ }^{25}$ R. Laenen, C. Rauscher, and A. Laubereau, Phys. Rev. Lett. 80, 2622 (1998).

${ }^{26}$ G. M. Gale, G. Gallot, F. Hache, N. Lascoux, S. Bratos, and J.-C. Leicknam, Phys. Rev. Lett. 82, 1068 (1999).

${ }^{27}$ S. Woutersen and H. J. Bakker, Phys. Rev. Lett. 83, 2077 (1999).

${ }^{28}$ J. Stenger, D. Madsen, P. Hamm, E. T. J. Nibbering, and T. Elsaesser, Phys. Rev. Lett. 87, 027401 (2001).

${ }^{29}$ E. T. J. Nibbering and T. Elsaesser, Chem. Rev. (Washington, D.C.) 104, 1887 (2004).

${ }^{30}$ M. L. Cowan, B. D. Bruner, N. Huse, J. R. Dwyer, B. Chugh, E. T. J. Nibbering, T. Elsaesser, and R. J. D. Miller, Nature (London) 434, 199 (2005).

${ }^{31}$ J. B. Asbury, T. Steinel, K. Kwak, S. A. Corcelli, C. P. Lawrence, J. L. Skinner, and M. D. Fayer, J. Chem. Phys. 121, 12431 (2004).

${ }^{32}$ J. J. Loparo, S. T. Roberts, and A. Tokmakoff, J. Chem. Phys. 125, 194522 (2006).

${ }^{33}$ A. Novak, Struct. Bonding (Berlin) 18, 177 (1974).

${ }^{34}$ W. Mikenda, J. Mol. Struct. 147, 1 (1986).

${ }^{35}$ S. Bratos, G. M. Gale, G. Gallot, F. Hache, N. Lascoux, and J.-C. Leicknam, Phys. Rev. E 61, 5211 (2000).

${ }^{36}$ H. J. Bakker, H.-K. Nienhuys, G. Gallot, N. Lascoux, G. M. Gale, J.-C. Leicknam, and S. Bratos, J. Chem. Phys. 116, 2592 (2002).

${ }^{37}$ PROPATHGroup. PROPATH: a program package for thermophysical properties, Version 12.1, 2001

${ }^{38}$ E. U. Franck and K. Roth, Discuss. Faraday Soc. 43, 108 (1967).

${ }^{39}$ Y. E. Gorbaty and A. G. Kalinichev, J. Phys. Chem. 99, 5336 (1995).

${ }^{40}$ T. Tassaing, Y. Danten, and M. Besnard, J. Mol. Liq. 101, 149 (2002).

${ }^{41}$ M. P. Allen and D. J. Tildesley, Computer Simulation of Liquids (Clarendon, Oxford, 1996).

${ }^{42}$ K. Refson, Comput. Phys. Commun. 126, 310 (2000).

${ }^{43}$ H. J. C. Berendsen, J. R. Grigera, and T. P. Straatsma, J. Phys. Chem. 91, 6269 (1987)

${ }^{44}$ C. P. Lawrence and J. L. Skinner, J. Chem. Phys. 118, 264 (2003).

${ }^{45}$ D. W. Oxtoby, D. Levesque, and J.-J. Weis, J. Chem. Phys. 68, 5528 (1978).

${ }^{46}$ R. Rey, K. B. Møller, and J. T. Hynes, J. Phys. Chem. A 106, 11993 (2002).

${ }^{47}$ C. P. Lawrence and J. L. Skinner, J. Chem. Phys. 117, 8847 (2002).

${ }^{48}$ D. Schwarzer, J. Lindner, and P. Vöhringer, J. Chem. Phys. 123, 161105 (2005).

${ }^{49}$ D. Schwarzer, J. Lindner, and P. Vöhringer, J. Phys. Chem. A 110, 2858 (2006). 